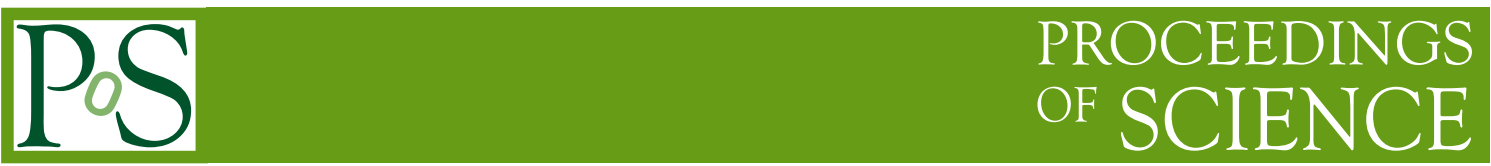

\title{
CPT and Decoherence in Quantum Gravity
}

\author{
Nikolaos E. Mavromatos*t \\ King's College London, Department of Physics, London, Strand WC2R 2LS, U.K. \\ E-mail: nikolaos.mavromatos@kcl.ac.uk
}

In this review, I first discuss briefly some theoretical motivations for potential Lorentz Violation and deviation from ordinary quantum mechanical behavior (decoherence) of field theoretic systems in the background of some quantum gravity (QG) models. Both types of effects lead to CPT violation, but they can be disentangled experimentally. I, then, proceed to a description of precision tests of CPT symmetry using neutral and charged Kaons, which are of direct relevance to the main theme of this conference. I emphasize the potentially unique rôle of neutral meson factories in providing "smoking-gun" evidence of some QG-decoherence models in which the CPT quantum mechanical operator is not well defined. This is achieved by means of potential observations of QG-induced modifications of the pertinent Einstein-Podolsky-Rosen (EPR) particle correlations.

Kaon International Conference

May 21-25, 2007

Laboratori Nazionali di Frascati dell'INFN

\footnotetext{
${ }^{*}$ Speaker.

${ }^{\dagger}$ Work partially supported by the European Union through the Marie Curie Research and Training Network UniverseNet MRTN-CT-2006-035863.
} 


\section{Lorentz Violation and Decoherence from Quantum Gravity: Motivations}

Any quantum theory, formulated on flat space times, is symmetric under the combined action of CPT transformations, provided the theory respects (i) Locality, (ii) Unitarity (i.e. conservation of probability) and (iii) Lorentz invariance. This is the celebrated CPT theorem [1]. An extension of this theorem to Quantum Gravity (QG) is by no means an obvious one; there may be information loss, in certain space-time foam backgrounds [2], implying an evolution from pure to mixed quantum states, and hence decoherence $[2,3]$. In such situations particle phenomenology has to be reformulated $[3,4]$ by viewing our low-energy world as an open quantum system. A similar situation might be encountered in Cosmologies with a cosmological constant, a model that seems to be favored by current astrophysical data on the acceleration of the Universe. Such models are characterized by cosmic (de Sitter) horizons, and again asymptotic states cannot be defined, and one may face a decoherence situation as a result of environmental degrees of freedom beyond the horizon (this issue however is still wide open, as the nature of the "microstates" of the de Sitter system is not understood at present). In all such cases the $\$$ matrix, connecting asymptotic in and out density matrices rather than pure-state wave vectors, $\rho_{\text {out }}=\$ \rho_{\text {in }}$ is not invertible, and this implies, by means of a theorem due to R. Wald [5], that the CPT operator itself is not well defined, at least from an effective field theory point of view. This is a strong form of CPT Violation (CPTV). This form of CPTV introduces a fundamental arrow of time/microscopic time irreversibility, unrelated in principle to CP properties. However, this arrow may not be observable experimentally, if the experimentalist has access to the so-called decoherence-free subspaces, which can be achieved, for instance, if the CPTV effects cancel out between particle and antiparticle sectors. This leads to a weak form of CPT invariance [5]. This is a model dependent statement, and therefore subject to experimental verification in principle. Within the scope of the present talk I will restrict myself to decoherence and CPT invariance tests within neutral Kaons $[3,6,7,8]$. This type of (decoherenceinduced) CPTV exhibits some fairly unique effects in $\phi(B$-meson, ...) factories [9], associated with a potential modification of the Einstein-Podolsky-Rosen (EPR) correlations of the entangled neutral Kaon $(B$-meson, $\ldots)$ states produced after the decay of the $\phi$-(or $\Upsilon-, \ldots)$ meson.

Another fundamental reason for CPTV in QG is the spontaneous breaking of Lorentz symmetry $(S B L)$ [10], without necessarily implying decoherence. In this case the ground state of the field theoretic system is characterized by non trivial vacuum expectation values of certain tensorial quantities, $\left\langle\mathscr{A}_{\mu}\right\rangle \neq 0$, or $\left\langle\mathscr{B}_{\mu_{1} \mu_{2} \ldots}\right\rangle \neq 0$. A concrete example of SBL may be provided by string field theory models of open bosonic strings [11]. In such models, there are cubic terms in an effective low-energy (target-space) Lagrangian involving the tachyonic scalar field $T$, that characterizes the bosonic string vacuum, and invariant products of higher-tensor fields that appear in the mode expansion of a string field, $T \mathscr{B}_{\mu_{1} \ldots \mu_{n}} \mathscr{B}^{\mu_{1} \ldots \mu_{n}}$. The negative mass squared tachyon field, then, acts as a Higgs field in such theories, acquiring a vacuum expectation value, which, in turn, implies non-zero vacuum expectation values for the tensor fields $\mathscr{B}$, leading in this way to energetically preferable configurations that are Lorentz Violating (LV). From the point of view of string theory landscape these are perfectly acceptable vacua [11]. An effective target-space field theory framework to discuss the phenomenology of such LV theories is the so-called Standard Model Extension (SME). For our purposes in this work, the upshot of SME is that there is a Modified Dirac Equation 
for spinor fields $\psi$, representing leptons and quarks with charge $q$ :

$$
\left(i \gamma^{\mu} D^{\mu}-M-a_{\mu} \gamma^{\mu}-b_{\mu} \gamma_{5} \gamma^{\mu}-\frac{1}{2} H_{\mu v} \sigma^{\mu v}+i c_{\mu \nu} \gamma^{\mu} D^{v}+i d_{\mu \nu} \gamma_{5} \gamma^{\mu} D^{v}\right) \psi=0
$$

where $D_{\mu}=\partial_{\mu}-A_{\mu}^{a} T^{a}-q A_{\mu}$ is an appropriate gauge-covariant derivative. The non-conventional terms proportional to the coefficients $a_{\mu}, b_{\mu}, c_{\mu \nu}, d_{\mu v}, H_{\mu v}, \ldots$, stem from the corresponding local operators of the effective Lagrangian which are phenomenological at this stage. The set of terms pertaining to $a_{\mu}, b_{\mu}$ entail CPT and Lorentz Violation, while the terms proportional to $c_{\mu v}, d_{\mu v}, H_{\mu v}$ exhibit Lorentz Violation only. It should be stressed that, within the SME framework (as also with the decoherence approach to QG), CPT violation does not necessarily imply mass differences between particles and antiparticles.

Some remarks are now in order, regarding the form and order-of-magnitude estimates of the Lorentz and/or CPT violating effects. In the approach of [10] the SME coefficients have been taken to be constants. Unfortunately there is not yet a detailed microscopic model available, which would allow for concrete predictions of their order of magnitude. Theoretically, the (dimensionful, with dimensions of energy) SME parameters can be bounded by applying renormalization group and naturalness assumptions to the effective local SME Hamiltonian, which leads to bounds on $b_{\mu}$ of order $10^{-17} \mathrm{GeV}$. At present all SME parameters should be considered as phenomenological and to be constrained by experiment. In general, however, the constancy of the SME coefficients may not be true. In fact, in certain string-inspired or stochastic models of space-time foam that violate Lorentz symmetry $[4,12]$, the coefficients $a_{\mu}, b_{\mu} \ldots$ are probe-energy $(E)$ dependent, as a result of back-reaction effects of matter onto the fluctuating space-time. Specifically, in stochastic models of space-time foam, one may find [12] that on average there is no Lorentz and/or CPT violation, i.e., the respective statistical v.e.v.s (over stochastic space-time fluctuations) $\left\langle a_{\mu}, b_{\mu}\right\rangle=0$, but this is not true for higher order correlators of these quantities (fluctuations), i.e., $\left\langle a_{\mu} a_{v}\right\rangle \neq 0,\left\langle b_{\mu} a_{v}\right\rangle \neq$ $0,\left\langle b_{\mu} b_{v}\right\rangle \neq 0, \ldots$. In such a case the SME effects will be much more suppressed, since by dimensional arguments such fluctuations are expected to be at most of order $E^{4} / M_{P}^{2}$, probably with no chance of being observed in immediate-future facilities, and certainly not in neutral kaon systems in the foreseeable future.

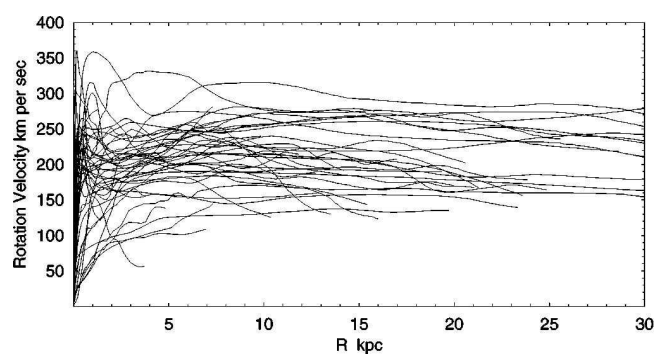

Figure 1: Collage of Rotational Curves of nearby spiral galaxies obtained by combining Doppler data from $\mathrm{CO}$ molecular lines for the central regions, optical lines for the disks, and $\mathrm{HI} 21 \mathrm{~cm}$ line for the outer (gas) disks. Graph from Y. Sophue and V. Rubin, Ann. Rev. Astron. Astrophys., Volume 31 (2001), 127.

We mention at this stage that LV theories have been recently invoked in cosmology as a way to bypass the dark matter problem, by providing relativistic field theories of gravity (TeVeS-like models) [13], with isotropic vector LV fields, $a_{\mu}(t)$, with only $a_{0}(t) \neq 0$, which are such that: 
(i) at galactic scales, and for small gravitational accelerations, $g \leq\left(200 \mathrm{~km} \mathrm{sec}^{-1}\right)^{2} /(10 \mathrm{kpc})$, they result in Modified Newtonian Dynamics of the type proposed in [14], leading to an experimental reproduction of the observed rotational curves of galaxies (see fig. 1), without the postulate of dark matter halos, and (ii) their vector-field instabilities exhibit [15] enhanced cosmic growth at galactic scales, in agreement with observations (see fig. 2), obviating once more the need for dark matter.

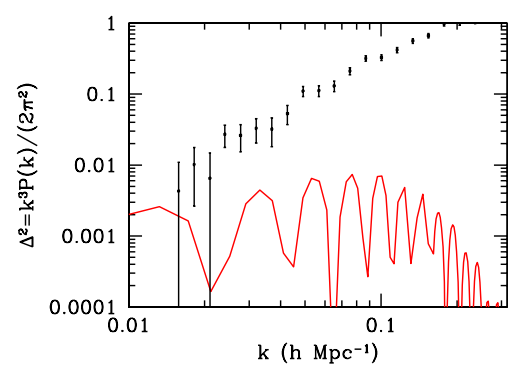

Figure 2: Power spectrum $\Delta^{2}=k^{3} P(k) /\left(2 \pi^{2}\right)$ vs. the scale $k$ of matter fluctuations (red curve, with wiggles) in a theory without dark matter as compared to observations of the galaxy power spectrum.

Although it is still unclear whether such models can fit all the available galactic and cosmological data, in particular data from the bullet cluster of galaxies (see fig. 3) and/or cosmic microwave background $(\mathrm{CMB})$ data, nevertheless there are recent claims that the CMB acoustic peaks could be fitted in the framework of such TeVeS-like models [16], provided that, in addition to the cosmological Lorentz Violation, hot dark matter of massive neutrinos (of order 15\%) is present. Thus the issue of cosmological Lorentz Violation may still be considered as open, which could be resolved by particle physics tests. The latter can in principle provide upper bounds for the LV effects, which then could be compared with the ones required by observational cosmology, in order for LV to play an alternative rôle to Dark Matter. In this talk I will restrict myself to Lorentz symmetry tests using neutral Kaons [10], and discuss the most recent bounds.

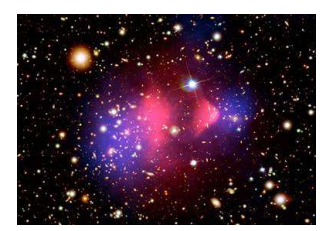

Figure 3: The Bullet cluster of Galaxies: the blue areas indicate Dark Matter inferred by Gravitational Lensing Techniques, whilst the red areas denote luminous matter detected by $\mathrm{x}$-rays. This galaxy provides an example where the Modified Newtonian Dynamics Theory might be in trouble.

I must stress at this point that QG-decoherence and Lorentz Violation are in principle independent [4]. The important difference of CPT violation in SBL models of Quantum Gravity from that in space-time foam situations lies on the fact that in the former case the CPT operator is well defined, but it does not commute with the effective Hamiltonian of the matter system. In such cases one may parametrize the Lorentz and/or CPT breaking terms by local field theory operators in the effective lagrangian, leading to a construction known as the "standard model extension" (SME) [10], which is a framework to study precision tests of such effects. Frame dependence is important in disentangling LV effects from Lorentz invariant models. In certain circumstances one 
may also violate locality, but I will not discuss this case explicitly here. Of course violations of locality could also be tested with high precision by means of a study of discrete symmetries in meson systems. I must stress that the phenomenology of CPT violation is complicated, and there seems not to be a single figure of merit for it. Depending on the precise way by which CPT violation is realized in a given class of models of QG, there are different ways by which we can test the violation [4]. I stress again that within the above frameworks, CPT violation does not necessarily imply mass differences between particles and antiparticles.

\section{Lorentz Violation and Neutral Kaons}

I commence the discussion by a very brief description of experimental tests of Lorentz symmetry, within the SME framework, using neutral Kaons, both single [10] and entangled states in a $\phi$ factory $[17,18]$. In order to isolate the terms in SME effective Hamiltonian that are pertinent to neutral Kaon tests, one should notice [10] that the relevant CPTV and LV parameter $\delta_{K}$ must be flavour diagonal, $\mathrm{C}$ violating but $\mathrm{P}, \mathrm{T}$ preserving, as a consequence of strong interaction properties in neutral meson evolution. This implies that $\delta_{K}$ is sensitive only to the $-a_{\mu}^{q} \bar{q} \gamma_{\mu} q$ quark terms in SME [10], where $a_{\mu}$ is a Lorentz and CPT violating parameter, with dimensions of energy, and $q$ denote quark fields, with the meson composition being denoted by $M=q_{1} \bar{q}_{2}$. The analysis of [10], then, leads to the following relation of the Lorentz and CPT violating parameter $a_{\mu}$ to the CPT violating parameter $\delta_{K}$ of the neutral Kaon system:

$$
\delta_{K} \simeq i \sin \widehat{\phi} \exp (i \widehat{\phi}) \gamma\left(\Delta a_{0}-\vec{\beta}_{K} \cdot \Delta \vec{a}\right) / \Delta m
$$

with the short-hand notation $S=$ =short-lived, $L=$ long-lived, $\Delta m=m_{L}-m_{S}, \Delta \Gamma=\Gamma_{S}-\Gamma_{L}, \widehat{\phi}=$ $\arctan (2 \Delta m / \Delta \Gamma), \quad \Delta a_{\mu} \equiv a_{\mu}^{q_{2}}-a_{\mu}^{q_{1}}$, and $\beta_{K}^{\mu}=\gamma\left(1, \vec{\beta}_{K}\right)$ the 4-velocity of the boosted kaon, with $\gamma$ the Lorentz factor. The experimental bounds of $a_{\mu}$ in neutral-Kaon experiments are based on searches of sidereal variations of $\delta_{K}$ (day-night effects), as in fig. 4.

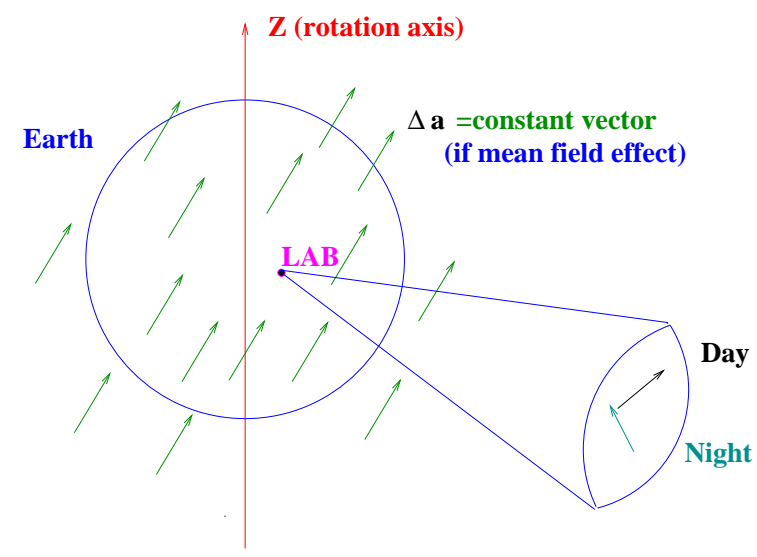

Figure 4: Schematic representation of searches for sidereal variations of the CPT-violating parameter $\delta_{K}$ in the SME framework. The green arrows, crossing the Earth indicate a constant Lorentz-violating vector that characterizes the Lorentz-violating ground state. 
From KTeV experiment [19] the following bounds of the $X$ and $Y$ components of the $a_{\mu}$ parameter have been obtained $\Delta a_{X}, \Delta a_{Y}<9.2 \times 10^{-22} \mathrm{GeV}$, where $X, Y, Z$ denote sidereal coordinates. Complementary measurements for the $a_{Z}$ component can come from $\phi$ factories [17].

In the case of $\phi$-factories there is additional dependence of the CPT-violating parameter $\delta_{K}$ on the polar $(\theta)$ and azimuthal $(\phi)$ angles

$$
\begin{aligned}
& \delta_{K}^{\phi}(|\vec{p}|, \theta, t)=\frac{1}{\pi} \int_{0}^{2 \pi} d \phi \delta_{K}(\vec{p}, t) \simeq i \sin \widehat{\phi} \exp (i \widehat{\phi})(\gamma / \Delta m) . \\
& \cdot\left(\Delta a_{0}+\beta_{K} \Delta a_{Z} \cos \chi \cos \theta+\beta_{K} \Delta a_{X} \sin \chi \cos \theta \cos (\Omega t)+\beta_{K} \Delta a_{Y} \sin \chi \cos \theta \sin (\Omega t)\right)
\end{aligned}
$$

where $\Omega$ denotes the Earth's sidereal frequency, and $\chi$ is the angle between the laboratory Z-axis and the Earth's axis. The experiment KLOE at DA $\Phi N E$ is sensitive to $a_{Z}$ : limits on $\delta\left(\Delta a_{Z}\right)$ can be placed from forward-backward asymmetry measurements $A_{L}=2 \operatorname{Re} \varepsilon_{K}-2 \operatorname{Re} \delta_{K}$. For more details on the relevant experimental bounds we refer the reader to the literature $[17,18]$. We only mention at this stage that in an upgraded DAФNE facility, namely experiment KLOE-2 at DAФNE-2, the expected sensitivity is [17] $\Delta a_{\mu}=\mathscr{O}\left(10^{-18}\right) \mathrm{GeV}$ which, although not competitive with the current $\mathrm{KTeV}$ limits on $a_{X, Y}$ given above, nevertheless constitutes an independent complementary measurement of the $a_{Z}$ component. Moreover, by looking at semileptonic decays, KLOE experiment can place limits (of order $10^{-18}$ ) on the time components $\Delta a_{0}$ of this Lorentz and CPT Violating parameter (A. DiDomenico, private communication), which, as we discussed in the previous section might be the only non zero component in an isotropic cosmological model of Lorentz Violation. Of course, it might well be that LV/CPTV effects cancel out between particle-antiparticle sectors, in which case they will be unobservable. This is a model dependent statement. Finally, I mention that other precision tests can be performed using other meson factories (B-mesons, etc.... ), which would also allow one to test the universality of QG Lorentz-violating effects, if observed.

\section{Quantum Gravity Decoherence and Neutral Kaons}

Quantum Gravity may induce decoherence and oscillations between Neutral-Kaon states $K^{0} \leftrightarrow$ $\bar{K}^{0}[3,6]$, thereby implying a two-level quantum mechanical system interacting with a QG "environment". Upon the general assumptions of average energy conservation and monotonic entropy increase, and the specific (to the Kaon system) assumption about the respect of the $\Delta S=\Delta Q$ rule by the QG medium, the modified evolution equation for the reduced density matrices, $\rho$, of the Neutral-Kaon matter reads [3]:

$$
\partial_{t} \rho=i[\rho, H]+\delta H \rho, \quad \delta H_{\alpha \beta}=\left(\begin{array}{cccc}
0 & 0 & 0 & 0 \\
0 & 0 & 0 & 0 \\
0 & 0 & -2 \alpha & -2 \beta \\
0 & 0 & -2 \beta & -2 \gamma
\end{array}\right) .
$$

where $H$ denotes the hamiltonian of the Kaon system, that may contain (possible) CPTV differences of masses and widths between particles and antiparticles [6], and $\delta \not H$ is the decoherence matrix. Positivity of $\rho$ requires: $\alpha, \gamma>0, \quad \alpha \gamma>\beta^{2}$. Notice that $\alpha, \beta, \gamma$ violate both CPT, due to their decoherening nature [5], and CP symmetry, as they do not commute with the CP operator $\widehat{C P}[6]: \widehat{C P}=\sigma_{3} \cos \theta+\sigma_{2} \sin \theta, \quad\left[\delta H_{\alpha \beta}, \widehat{C P}\right] \neq 0$. As pointed out in [8], however, in the case 
of $\phi$-factories complete positivity is guaranteed within the above (single-particle) framework only if the further conditions $\alpha=\gamma$ and $\beta=0$ are imposed. Experimentally the complete positivity hypothesis, and thus the above framework, can be tested explicitly by keeping all three parameters. In what follows, as far as single Kaon states are concerned, we shall keep the $\alpha, \beta, \gamma$ parametrization [6], and give the available experimental bounds for these parameters. The relevant observables

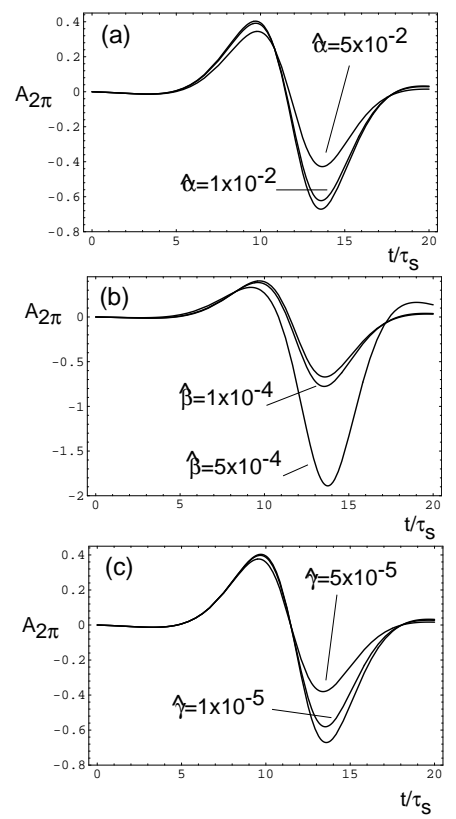

Figure 5: Typical neutral kaon decay asymmetries $A_{2 \pi}[6]$ indicating the effects of QG induced decoherence.

are defined as $\left\langle O_{i}\right\rangle=\operatorname{Tr}\left[O_{i} \rho\right]$. One looks at the time evolution of decay asymmetries [6] (see fig. 5 for the case of $2 \pi$ final states). The important point to notice is that the two types of CPTV, within and outside quantum mechanics, can be disentangled experimentally [6]. We next mention that, typically, for instance when the final states are $2 \pi$, one has a time evolution of the decay rate $R_{2 \pi}: R_{2 \pi}(t)=c_{S} e^{-\Gamma_{S} t}+c_{L} e^{-\Gamma_{L} t}+2 c_{I} e^{-\Gamma t} \cos (\Delta m t-\phi)$, where $S=$ short-lived, $L=$ long-lived, $I=$ interference term, $\Delta m=m_{L}-m_{S}, \Delta \Gamma=\Gamma_{S}-\Gamma_{L}, \Gamma=\frac{1}{2}\left(\Gamma_{S}+\Gamma_{L}\right)$. One may thus define the Decoherence Parameter $\zeta=1-\frac{c_{I}}{\sqrt{c_{S} C_{L}}}$, as a (phenomenological) measure of quantum decoherence induced in the system. In our decoherence scenario, $\zeta$ corresponds to a particular combination of the decoherence parameters [6] $\zeta \rightarrow \frac{\widehat{\gamma}}{2\left|\varepsilon^{2}\right|}-2 \frac{\widehat{\beta}}{|\varepsilon|} \sin \phi$, with $\widehat{\gamma}=\gamma / \Delta \Gamma$, etc. The CPLEAR measurements gave the following bounds [20] $\alpha<4.0 \times 10^{-17} \mathrm{GeV},|\beta|<2.3 . \times 10^{-19} \mathrm{GeV}, \gamma<$ $3.7 \times 10^{-21} \mathrm{GeV}$, which are not much different from theoretically expected values in some optimistic scenarios [6] $\alpha, \beta, \gamma=O\left(\frac{E^{2}}{M_{P}}\right)$. The experiment KLOE at DaФNE updated these limits recently by measuring for the first time the $\gamma$ parameter for entangled Kaon states $[17,18]$ : $\gamma_{\mathrm{KLOE}}=\left(1.1_{-2.4}^{+2.9} \pm 0.4\right) \times 10^{-21} \mathrm{GeV}$, as well as the (naive) decoherence parameter $\zeta$. This bound can be improved by an order of magnitude in upgraded facilities, such as KLOE-2 [17].

\section{Decoherence-CPTV and Modified EPR Correlations of Entangled Neutral Kaons}

If CPT is intrinsically violated, in the sense of being not well defined due to decoherence [5], the Neutral mesons $K^{0}$ and $\bar{K}^{0}$ should no longer be treated as identical particles. As a conse- 
quence [9], the initial entangled state in $\phi$ factories $\mid i>$, after the $\phi$-meson decay, reads:

$\mid i>=\mathscr{N}\left[\left(\left|K_{S}(\vec{k}), K_{L}(-\vec{k})>-\right| K_{L}(\vec{k}), K_{S}(-\vec{k})>\right)+\omega\left(\left|K_{S}(\vec{k}), K_{S}(-\vec{k})>-\right| K_{L}(\vec{k}), K_{L}(-\vec{k})>\right)\right]$

where $\omega=|\omega| e^{i \Omega}$ is a complex parameter, parametrizing the intrinsic CPTV modifications of the EPR correlations (" $\omega$-effect”). The $\omega$-parameter controls the amount of contamination of the final $\mathrm{C}$ (odd) state by the "wrong" (C(even)) symmetry state. The appropriate observable (see fig. 6) is the "intensity" $I(\Delta t)=\int_{\Delta t \equiv\left|t_{1}-t-2\right|}^{\infty}|A(X, Y)|^{2}$, with $A(X, Y)$ the appropriate $\phi$ decay amplitude [9], where one of the Kaon products decays to the final state $X$ at $t_{1}$ and the other to the final state $Y$ at time $t_{2}$ (with $t=0$ the moment of the $\phi$ decay).

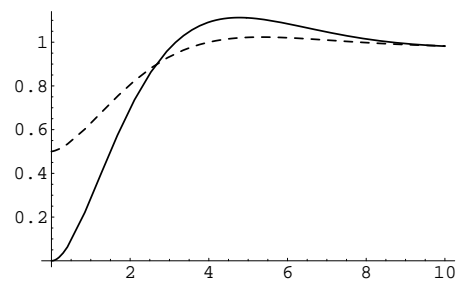

Figure 6: A characteristic case of the intensity $I(\Delta t)$, with $|\omega|=0$ (solid line), vs $\Delta t$, compared with (dashed line) $I(\Delta t)$, with $|\omega|=\left|\eta_{+-}\right|, \Omega=\phi_{+-}-0.16 \pi$, for definiteness [9].

The KLOE experiment at DAФNE has released the first measurement of the $\omega$ parameter $[17,18]: \operatorname{Re}(\omega)=\left(1.1_{-5.3}^{+8.7} \pm 0.9\right) \times 10^{-4}, \operatorname{Im}(\omega)=\left(3.4_{-5.0}^{+4.8} \pm 0.6\right) \times 10^{-4}$. At least an order of magnitude improvement is expected for upgraded facilities such as KLOE-2 at (the upgraded) DAФNE-2 [17]. This sensitivity is not far from certain optimistic models of space time foam leading to $\omega$-like effects [12]. The $\omega$ effect can be disentangled experimentally from both, the $\mathrm{C}$ (even) background - by means of different interference with the $\mathrm{C}$ (odd) resonant contributions, and the decoherent evolution $(\alpha=\gamma)$ effects [9] - due to different structures. Finally, I close this section by mentioning that, if this type of intrinsic CPT violation is due to a LV decoherent model, then this should show up in a different size of the effect (if observed) in B-factories, where the products of the decay of the $\Upsilon$-meson are boosted as compared to those of the decay of the $\phi$-meson in $\phi$ factories, which occurs at rest. As far as $B$-factories are concerned, I also mention that the $\omega$-effect leads to intrinsic limitations for the accuracy of flavor tagging [21].

\section{Precision T, CP and CPT Tests with Charged Kaons}

Precision tests of discrete symmetries can also be performed with charged Kaons, as a result of the (recently acquired) high statistics at the NA48 experiment [22], in certain decay channels, for instance $K^{ \pm} \rightarrow \pi^{+}+\pi^{-}+\ell^{ \pm}+v_{\ell}\left(\bar{v}_{\ell}\right)$, abbreviated as $K_{\ell 4}^{ \pm}$. One can perform independent precision tests of T, CP and CPT using this reaction [23], by comparing the decay rates of the $K^{+}$ mode with the corresponding decays of the $K^{-}$mode, as well as tests of $\Delta S=\Delta Q$ and $|\Delta I|=1 / 2$ isospin rules. If CPT is violated, through microscopic time irreversibility [5], then the phase space analysis for the products of the reaction, from which one obtains the di-pion strong-interaction phase shifts, needs to be modified [23]. I would like to finish by mentioning the possibility of exploiting the recently attained high statistics for charged Kaons in the NA48 experiment [22] so 
as to use appropriate combinations of both reaction modes $K_{\ell 4}^{ \pm}$for precision tests of physics beyond the Standard Model (SM), such as supersymmetry, etc., including possible CPT violations. One could look at T-odd triple momentum correlators [24]: $\vec{p}_{\ell} \cdot\left(\vec{p}_{\pi_{1}} \times \vec{p}_{\pi_{2}}\right)$. The so constructed CPviolating observables are independent of the lepton polarization and thus easier to measure in a high statistics environment, such as the NA48 experiment [22].

\section{References}

[1] G. Lüders, Ann. Phys. (NY) 2 (1957) 1.

[2] J.A.Wheeler and K.Ford, Geons,Black Holes and Quantum Foam: A Life In Physics, (New York, USA: Norton (1998)); S.W.Hawking, Comm. Math. Phys. 87 (1982) 395.

[3] J.R. Ellis et al., Nucl. Phys. B 241 (1984) 381.

[4] N.E. Mavromatos, Lect. Notes Phys. 669(2005) 245 [gr-qc/0407005] and references therein; J. Bernabeu et al., arXiv:hep-ph/0607322, III Da $\Phi$ NE Physics Handbook, in press.

[5] R. Wald, Phys. Rev. D21 (1980) 2742.

[6] J.R. Ellis, N.E. Mavromatos, D.V.Nanopoulos, Phys. Lett. B 293 (1992) 142; J.R. Ellis et al. Phys. Rev. D 53 (1996) 3846.

[7] P.Huet and M.E.Peskin, Nucl. Phys. B 434 (1995) 3.

[8] F.Benatti and R.Floreanini, Nucl. Phys. B 511 (1998) 550.

[9] J.Bernabeu, N.E. Mavromatos, J.Papavassiliou, Phys. Rev. Lett. 92 (2004) 131601; J. Bernabeu et al., Nucl. Phys. B 744 (2006) 180.

[10] V.A.Kostelecky, CPT and Lorentz Symmetry III (World Scientific, Singapore 2005); Phys. Rev. Lett. 80 (1998) 1818.

[11] V. A. Kostelecky and R. Potting, Nucl. Phys. B 359 (1991) 545; Phys. Lett. B 381 (1996) 89.

[12] J. Bernabeu, N. E. Mavromatos and Sarben Sarkar, Phys. Rev. D 74 (2006) 045014.

[13] J. D. Bekenstein, Phys. Rev. D 70 (2004) 083509 [Erratum-ibid. D 71 (2005) 069901].

[14] M. Milgrom, Astrophys. J. 270 (1983) 365.

[15] S. Dodelson and M. Liguori, Phys. Rev. Lett. 97 (2006) 231301.

[16] C. Skordis et al., Phys. Rev. Lett. 96 (2006) 011301.

[17] F. Ambrosino et al. [KLOE Collaboration], Phys. Lett. B 642 (2006) 315; A. Di Domenico, Mini Workshop on Neutral Kaon Interferometry, Frascati LN, March 242006 (http://www.roma1.infn.it/people/didomenico/roadmap/kaoninterferometry.html)

[18] M. Testa, these proceedings.

[19] M.J. Wilking, arXiv:hep-ex/0606044.

[20] R. Adler et al. [CPLEAR collaboration], Phys. Lett. B 364 (1995) 239.

[21] E. Alvarez et al., Phys. Lett. B 607 (2005) 197.

[22] C. Lazzeroni [NA48 Collaboration], PoS HEP2005 (2006) 096.

[23] T.D. Lee and C.S. Wu, Ann. Review Nuclear Science, Vol. 16 (1966) 471; A. Pais and S.B. Treiman, Phys. Rev. 168 (1968) 1858.

[24] A. Retico, Phys. Rev. D 65 (2002) 117901. 